\title{
AMOSTRAGEM PRELIMINAR DA MASTOFAUNA EM VEGETAÇÃO REMANESCENTE DE MATA ATLÂNTICA

\author{
Caio Ferreira ${ }^{1}$ \\ Douglas Pereira Lima Gomes² \\ Andréa Chaguri ${ }^{3}$ \\ Nádia Maria Rodrigues de Campos Velho ${ }^{4}$ \\ Karla Andressa Ruiz Lopes ${ }^{5}$
}

\begin{abstract}
Resumo: Os mamíferos desempenham um papel fundamental no ecossistema como o controle populacional e a regeneração de matas. Buscou-se com este trabalho, obter uma amostragem preliminar da mastofauna presente em um remanescente de floresta atlântica, utilizando-se de armadilha fotográfica, contribuindo com maiores informações a respeito do uso de habitat por estes animais na área de estudo, local característico de um corredor ecológico, devido a sua paisagem ser bem semelhante ao mesmo. Foram obtidos registros de seis espécies de mamíferos silvestres pertencentes a quatro famílias e três ordens: quatro Carnivora (Cerdocyon thous, Lontra longicaudis, Galictis cuja, Canis lupus familiaris), um Didelphimorphia (Didelphis aurita) e um Rodentia (Hydrochoerus hydrochaeris), sendo que não foi possível a identificação de uma espécie. Ressalta-se a importância da presença da espécie Lontra longicaudis, que possui o estado de conservação quase ameaçada no estado de São Paulo.
\end{abstract}

Palavras-chave: Mamíferos; Fragmentação; Armadilha fotográfica; Corredor ecológico.

\footnotetext{
1 Universidade do Vale do Paraíba, Brasil. E-mail: cfvision09@gmail.com.

2 Universidade do Vale do Paraíba, Brasil. E-mail: douglasplgomes@yahoo.com.br.

3 Universidade do Vale do Paraíba, Brasil. E-mail: andreachaguri@gmail.com.

${ }^{4}$ Universidade do Vale do Paraíba, Brasil. E-mail: nvelho@univap.br.

5 Universidade do Vale do Paraíba, Brasil. E-mail: karla@univap.br.
} 